\title{
Positive solutions to singular Dirichlet-type boundary value problems of nonlinear fractional differential equations
}

\author{
Jiqiang Jiang ${ }^{1 *} \mathbb{D}$, Weiwei Liu and Hongchuan Wang ${ }^{1}$
}

"Correspondence: qfjjq@163.com 'School of Mathematical Sciences, Qufu Normal University, Qufu,

People's Republic of China

\begin{abstract}
In this paper, by using the properties of the Green function, $u_{0}$-positive operator and Gelfand's formula, some properties of the first eigenvalue corresponding to the relevant operator are obtained. Based on these properties, the fixed point index of the nonlinear operator is calculated explicitly and some sufficient conditions for the existence and uniqueness results of positive solution are established.
\end{abstract}

MSC: $34 \mathrm{~A} 08 ; 34 \mathrm{~B} 18$

Keywords: Positive solutions; First eigenvalue; $u_{0}$-positive operator; Singularity; Fixed point index

\section{Introduction}

The boundary value problems (BVPs) for fractional differential equations arise from the studies of models of aerodynamics, fluid flows, electrodynamics of complex medium, electrical networks, rheology, polymer rheology, economics, biology chemical physics, control theory, signal and image processing. Recently, the study of such kind of problems has received considerable attention both in theory and applications, see [1-30] and the references therein, such as resonant BVP $[3,18,25]$, singular BVP $[7,15,20,30]$, nonlocal BVP $[8,14,17,29]$ and semipositone BVP $[11,16,24,28]$.

Specially, there are a few papers considering the Dirichlet-type problem for ordinary differential equations of fractional order; see $[1,4,6,10,13,27]$. Bai and Lü [4] have investigated the following BVP:

$$
\left\{\begin{array}{l}
D_{0+}^{\alpha} x(t)+f(t, x(t))=0, \quad 0<t<1, \\
x(0)=0, \quad x(1)=0,
\end{array}\right.
$$

where $1<\alpha \leq 2$ and $f:[0,1] \times[0, \infty) \rightarrow[0, \infty)$ continuous, by means of the Krasnosel'skii fixed point theorem and the Leggett-Williams fixed point theorem, the existence and multiplicity of positive solutions for BVP (1.1) are obtained. By using the Krasnosel'skii fixed point theorem, Jiang and Yuan [13] studied the existence and multiplicity of positive solutions of BVP (1.1). By using the Leray-Schauder nonlinear alternative and a fixed point theorem on cones, BVP (1.1) are also studied in [27], the authors establish the exis-

(c) The Author(s) 2018. This article is distributed under the terms of the Creative Commons Attribution 4.0 International License (http://creativecommons.org/licenses/by/4.0/), which permits unrestricted use, distribution, and reproduction in any medium, provided you give appropriate credit to the original author(s) and the source, provide a link to the Creative Commons license, and indicate if changes were made. 
tence of multiple positive solutions to positone and semipositone Dirichlet-type BVP (1.1). In [10], the authors investigated problems (1.1) with $f(t, x(t))$ replaced by $\lambda a(t) f(t, x(t))$, the existence of positive solutions are obtained by means of the fixed point theory in cones.

Recently, in [26], the authors investigated the existence and uniqueness of the positive solutions for the two-term fractional differential equations of the type:

$$
\left\{\begin{array}{l}
-D_{0+}^{\alpha} x(t)+b x(t)=f(t, x(t)), \quad 0<t<1, \\
x(0)=0, \quad x(1)=0
\end{array}\right.
$$

where $1<\alpha<2, b>0, D_{0_{+}}^{\alpha}$ is the Riemann-Liouville fractional derivative. They derived the corresponding Green function called the fractional Green function and obtained some properties. By means of the mixed monotone operator theorem, the existence and uniqueness of positive solution are obtained under the singular conditions.

Inspired by the above work, we study the existence and uniqueness of the positive solutions for the following two-term fractional differential equations BVP:

$$
\left\{\begin{array}{l}
-D_{0+}^{\alpha} x(t)+b x(t)=a(t) f(t, x(t)), \quad 0<t<1, \\
x(0)=0, \quad x(1)=0,
\end{array}\right.
$$

where $1<\alpha<2, b>0, D_{0+}^{\alpha}$ is the Riemann-Liouville fractional derivative, $f:[0,1] \times$ $[0, \infty) \rightarrow[0, \infty)$ is continuous, $a(t)$ is continuous and may be singular at $t=0,1$. Under some certain conditions, by use of $u_{0}$-positive operator and the fixed poind index theorem, we obtain some existence and unique results of positive solutions.

Our work presented in this paper has the following features. First of all, we discuss the equation contain two term, i.e., $-D_{0_{+}}^{\alpha} x(t)+b x(t)=f(t, x(t))$, this is different from $[4,10]$, in other words, Ref. $[4,10]$ and this article discuss different problems. The second new feature is that BVP (1.2) possesses a singularity, that is, the nonlinear term may be singular at $t=0,1$; this is different from $[4,10]$. Thirdly, we discuss BVP $(1.2)$, by using the properties of the Green function, $u_{0}$-positive operator and Gelfand's formula, some sufficient conditions for the existence and uniqueness results of positive solution are established. The method used differs significantly from $[4,10,26]$. So our results are new and meaningful.

The rest of this paper is organized as follows. In Sect. 2, we present some lemmas that are used to prove our main results. In Sect. 3, under the assumption that $f(t, y)$ is a Lipschitz continuous function, by use of $u_{0}$-positive operator, the uniqueness of positive solutions is established. The interesting point is that the Lipschitz constant is related to the first eigenvalues corresponding to the relevant operators. In Sect. 4, the existence results of positive solutions are obtained by use of the fixed poind index and spectral radius of some related linear operator.

\section{Basic definitions and preliminaries}

For the convenience of the reader, we present here some notation and lemmas which will be used in the proofs of our results. 
Definition 2.1 The Riemann-Liouville fractional integral of order $\alpha>0$ of a function $u:(0, \infty) \rightarrow \mathbb{R}$ is given by

$$
I_{0+}^{\alpha} u(t)=\frac{1}{\Gamma(\alpha)} \int_{0}^{t}(t-s)^{\alpha-1} u(s) d s
$$

provided that the right-hand side is pointwise defined on $(0, \infty)$.

Definition 2.2 The Riemann-Liouville fractional derivative of order $\alpha>0$ of a function $u:(0, \infty) \rightarrow \mathbb{R}$ is given by

$$
\mathcal{D}_{0+}^{\alpha} u(t)=\frac{1}{\Gamma(n-\alpha)}\left(\frac{d}{d t}\right)^{n} \int_{0}^{t}(t-s)^{n-\alpha-1} u(s) d s,
$$

where $n=[\alpha]+1,[\alpha]$ denotes the integer part of number $\alpha$, provided that the right-hand side is pointwise defined on $(0, \infty)$.

Set

$$
G(t, s)=\frac{1}{H(1)} \begin{cases}H(t) H(1-s), & 0 \leq t \leq s \leq 1, \\ H(t) H(1-s)-H(t-s) H(1), & 0 \leq s \leq t \leq 1,\end{cases}
$$

where $H(t)=t^{\alpha-1} E_{\alpha, \alpha}\left(b t^{\alpha}\right)$, and

$$
E_{\alpha, \alpha}(y)=\sum_{k=0}^{+\infty} \frac{y^{k}}{\Gamma((k+1) \alpha)}
$$

is the Mittag-Leffler function [19, 21]. Denote

$$
h(y)=\frac{\alpha-2}{\Gamma(\alpha-1)}+\sum_{k=1}^{+\infty} \frac{y^{k}}{\Gamma((k+1) \alpha-2)}, \quad y \in[0, \infty) .
$$

It is easy to verify that there exists a unique $\tilde{b}>0$ such that $h(\tilde{b})=0$. We list the following assumptions adopted in this paper.

$\left(\mathrm{H}_{0}\right) b \in(0, \tilde{b}]$.

$\left(\mathrm{H}_{1}\right) f:[0,1] \times[0, \infty) \rightarrow[0, \infty)$ is continuous.

$\left(\mathrm{H}_{2}\right) a:(0,1) \rightarrow[0, \infty)$ is continuous and not identical zero on any closed subinterval of $(0,1)$ with

$$
0<\int_{0}^{1} s^{\alpha-j}(1-s)^{\alpha-j} a(s) d s<+\infty, \quad j=1,2 .
$$

The following three lemmas can be found in [26].

Lemma 2.3 Suppose that $\left(\mathrm{H}_{0}\right)$ holds and $\omega \in L[0,1]$. Then the problem

$$
\left\{\begin{array}{l}
-D_{0+}^{\alpha} x(t)+b x(t)=\omega(t), \quad 0<t<1 \\
x(0)=0, \quad x(1)=0
\end{array}\right.
$$


has a unique solution

$$
x(t)=\int_{0}^{1} G(t, s) \omega(s) d s .
$$

Lemma 2.4 Suppose that $\left(\mathrm{H}_{0}\right)$ holds, then the function $G(t, s)$ has the following properties:

(1) $G(t, s)>0, \forall t, s \in(0,1)$;

(2) $G(t, s)=G(1-s, 1-t), \forall t, s \in[0,1]$;

(3) $G(t, s) \leq H(1) s(1-s)^{\alpha-1} t^{\alpha-2}, \forall t, s \in[0,1]$;

(4) $G(t, s) \geq \Lambda s(1-s)^{\alpha-1}(1-t) t^{\alpha-1}, \forall t, s \in[0,1]$, where

$$
\Lambda=\min \left\{(\alpha-1)^{2} H(1), \frac{1}{H(1)(\Gamma(\alpha))^{2}}\right\}
$$

Lemma 2.5 The function $K(t, s)=: t^{2-\alpha} G(t, s)$ has the following properties:

(1) $K(t, s)>0, \forall t, s \in(0,1)$;

(2) $K(t, s) \leq H(1)(1-s)^{\alpha-2} t(1-t), \forall t, s \in[0,1]$;

(3) $K(t, s) \leq H(1) s(1-s)^{\alpha-1}, \forall t, s \in[0,1]$;

(4) $K(t, s) \geq \Lambda s(1-s)^{\alpha-1} t(1-t), \forall t, s \in[0,1]$.

Let $E=C[0,1]$, then $E$ is a Banach space with the norm $\|x\|=\max _{0 \leq t \leq 1}|x(t)|$, for any $x \in E$. Set $P=\{x \in E: x(t) \geq 0$ for $t \in[0,1]\}$. $P$ is a positive cone in $E$.

Define a nonlinear operator $T$ and a linear operator $L$ as follows:

$$
\begin{aligned}
& (T x)(t)=\int_{0}^{1} K(t, s) a(s) f\left(s, s^{\alpha-2} x(s)\right) d s, \quad x \in C[0,1] \\
& (L x)(t)=\int_{0}^{1} K(t, s) a(s) s^{\alpha-2} x(s) d s, \quad x \in C[0,1] .
\end{aligned}
$$

It is not difficult to verify that $L: E \rightarrow E$ is linear completely continuous and $L(P) \subset P$.

The following concept is due to Krasnosel'skii [31].

Definition 2.6 We say that a bounded linear operator $L: E \rightarrow E$ is $u_{0}$-positive on the cone $P$ if there exists $u_{0} \in P \backslash\{\theta\}$ such that for every $x \in P \backslash\{\theta\}$ there exist a natural number $n$ and positive constants $\beta(x), \gamma(x)$ such that

$$
\beta(x) u_{0} \leq L^{n} x \leq \gamma(x) u_{0}
$$

Lemma 2.7 L is $u_{0}$-positive operator with $u_{0}(t)=t(1-t)$.

Proof For any $x \in P \backslash\{\theta\}$, it follows from Lemma 2.5 that

$$
(L x)(t)=\int_{0}^{1} K(t, s) a(s) s^{\alpha-2} x(s) d s \leq H(1) t(1-t) \int_{0}^{1}(1-s)^{\alpha-2} a(s) s^{\alpha-2} x(s) d s .
$$

On the other hand, by Lemma 2.5, we also have

$$
(L x)(t)=\int_{0}^{1} K(t, s) a(s) s^{\alpha-2} x(s) d s \geq \Lambda t(1-t) \int_{0}^{1} s^{\alpha-1}(1-s)^{\alpha-1} a(s) x(s) d s .
$$

The above inequalities show that $L$ is $u_{0}$-positive operator with $u_{0}(t)=t(1-t)$. 
Lemma 2.8 (Krein-Rutmann [32]) Let $L: E \rightarrow E$ be a continuous linear operator, $P$ be a total cone and $L(P) \subset P$. If there exist $\psi \in E \backslash(-P)$ and a positive constant $c$ such that $c L(\psi) \geq \psi$, then the spectral radius $r(L) \neq 0$ and $L$ has a positive eigenfunction corresponding to its first eigenvalue $\lambda=(r(L))^{-1}$.

It follows from Lemma 2.7 and Lemma 2.8 that the spectral radius $r(L) \neq 0$, moreover $L$ has a positive eigenfunction $\varphi^{*}(t)$ corresponding to its first eigenvalue $\lambda_{1}=(r(L))^{-1}$.

Remark 2.9 Let $\varphi^{*}$ be the positive eigenfunction of $L$ corresponding to $\lambda_{1}$, i.e., $\lambda_{1} L \varphi^{*}=\varphi^{*}$. Then, by Definition 2.6 and Lemma 2.7, there exist $\delta_{1}\left(\varphi^{*}\right), \delta_{2}\left(\varphi^{*}\right)>0$ such that

$$
\delta_{1}\left(\varphi^{*}\right) u_{0} \leq L \varphi^{*}=\frac{1}{\lambda_{1}} \varphi^{*} \leq \delta_{2}\left(\varphi^{*}\right) u_{0} .
$$

Thus we found that $L$ is $\varphi^{*}$-positive operator.

Lemma 2.10 ([31]) Let $L$ be a completely continuous $u_{0}$-bounded operator, $\lambda_{1}>0$ is the first eigenvalue of $L, y_{0}$ is a positive eigenfunction which belongs to $P \backslash\{\theta\}$. Then, for any $y \in P \backslash\{\theta\}$ with $y \neq \mu y_{0}(\mu \geq 0), \lambda_{1} L y \nless y$ and $\lambda_{1} L y \ngtr y$.

\section{Uniqueness of positive solution}

We now wish to show that under certain conditions, BVP (1.2) has a unique positive solution.

Theorem 3.1 Suppose that $\left(\mathrm{H}_{0}\right),\left(\mathrm{H}_{1}\right)$ and $\left(\mathrm{H}_{2}\right)$ hold and there exists $\kappa \in[0,1)$ such that

$$
|f(t, u)-f(t, v)| \leq \kappa \lambda_{1}|u-v|, \quad \forall t \in[0,1], u, v \in[0, \infty)
$$

where $\lambda_{1}$ is the first eigenvalue of $L$ defined by (2.3). Then BVP (1.2) has a unique positive solution $\bar{x}=t^{\alpha-2} x^{*}$ in $P$, and for any $x_{0} \in P \backslash\{\theta\}$, the iterative sequence $x_{n}=T x_{n-1}(n=$ $1,2, \ldots)$ converges to $x^{*}$.

Proof It is not difficult to prove that $T: P \rightarrow P$ is completely continuous and the existence of positive solution $t^{\alpha-2} x$ for BVP (1.2) is equivalent to that of fixed point $x$ of $T$ in $P$.

For any given $x_{0} \in P \backslash\{\theta\}$, let $x_{n}=T x_{n-1}(n=1,2, \ldots)$. By Remark 2.9, there exists $\delta=$ $\delta\left(\left|x_{1}-x_{0}\right|\right)>0$ such that

$$
\left(L\left|x_{1}-x_{0}\right|\right)(t) \leq \delta \varphi^{*}(t), \quad t \in[0,1]
$$

For $m \in \mathbb{N}$,

$$
\begin{aligned}
& \left|x_{m+1}(t)-x_{m}(t)\right| \\
& \quad=\left|\left(T x_{m}\right)(t)-\left(T x_{m-1}\right)(t)\right| \\
& \quad=\left|\int_{0}^{1} K(t, s) a(s) f\left(s, s^{\alpha-2} x_{m}(s)\right) d s-\int_{0}^{1} K(t, s) a(s) f\left(s, s^{\alpha-2} x_{m-1}(s)\right) d s\right| \\
& \quad \leq \int_{0}^{1} K(t, s) a(s)\left|f\left(s, s^{\alpha-2} x_{m}(s)\right)-f\left(s, s^{\alpha-2} x_{m-1}(s)\right)\right| d s
\end{aligned}
$$




$$
\begin{aligned}
& \leq \kappa \lambda_{1} L\left(\left|x_{m}-x_{m-1}\right|\right)(t) \leq \cdots \leq \lambda_{1}^{m} \kappa^{m} L^{m}\left(\left|x_{1}-x_{0}\right|\right)(t) \\
& \leq \lambda_{1}^{m} \kappa^{m} L^{m-1}\left(\delta \varphi^{*}(t)\right)=\lambda_{1}^{m} \kappa^{m} \delta L^{m-1}\left(\varphi^{*}(t)\right) \\
& =\lambda_{1} \delta \kappa^{m} \varphi^{*}(t), \quad t \in[0,1] .
\end{aligned}
$$

So, for any $n, m \in \mathbb{N}$, we have

$$
\begin{aligned}
\left|x_{n+m+1}(t)-x_{n}(t)\right| & =\left|x_{n+m+1}(t)-x_{n+m}(t)+\cdots+x_{n+1}(t)-x_{n}(t)\right| \\
& \leq\left|x_{n+m+1}(t)-x_{n+m}(t)\right|+\cdots+\left|x_{n+1}(t)-x_{n}(t)\right| \\
& \leq \lambda_{1} \delta\left(\kappa^{m+n}+\cdots+\kappa^{n}\right) \varphi^{*}(t) \\
& =\lambda_{1} \delta \frac{\kappa^{n}\left(1-\kappa^{m+1}\right)}{1-\kappa} \varphi^{*}(t), \quad t \in[0,1] .
\end{aligned}
$$

Consequently,

$$
\left\|x_{n+m+1}-x_{n}\right\| \leq \lambda_{1} \delta \frac{\kappa^{n}\left(1-\kappa^{m+1}\right)}{1-\kappa}\left\|\varphi^{*}\right\| \rightarrow 0, \quad \text { as } n, m \rightarrow \infty .
$$

By the completeness of $P$, there exists a $x^{*} \in P$ such that $\lim _{n \rightarrow \infty} x_{n}=x^{*}$. Passing to the limit into $x_{n+1}=T x_{n}$ and using the fact that $T$ is continuous, it follows that $x^{*}$ is a fixed point of $T$ in $P$.

Now we show that $T$ has at most one fixed point in $P$. Suppose there exist two elements $x, y \in P$ with $x=T x$ and $y=T y$. By Lemma 2.7, there exists $\delta=\delta(|x-y|)>0$ such that

$$
(L|x-y|)(t) \leq \delta \varphi^{*}(t), \quad t \in[0,1]
$$

Then, for any $n \in \mathbb{N}$, we have

$$
|x(t)-y(t)|=\left|\left(T^{n} x\right)(t)-\left(T^{n} y\right)(t)\right| \leq \kappa^{n} \delta \lambda_{1} \varphi^{*}(t)
$$

which can happen only if $x=y$. This implies that $T$ has at most one fixed point.

Then $x^{*}$ is the unique fixed point of $T$ in $P$ and $\bar{x}=t^{\alpha-2} x^{*}$ is the unique positive solution of BVP (1.2). The proof is completed.

Remark 3.2 Let $m \rightarrow+\infty$ in (3.1), we have error estimation

$$
\left\|x_{n}-x^{*}\right\| \leq \lambda_{1} \delta \frac{\kappa^{n}}{1-\kappa}\left\|\varphi^{*}\right\|
$$

and with the rate of convergence

$$
\left\|x_{n}-x^{*}\right\|=O\left(\kappa^{n}\right)
$$

Remark 3.3 In Theorem 3.1 and Remark 3.2, we not only give the condition of the existence of a unique positive solution, but also establish an iterative sequence of solution and error estimation. In particular, since $u_{0}(t)=t(1-t) \in P$, and the initial value of the iterative sequence can begin from $x_{0}=u_{0}=t(1-t)$, this is simpler and helpful for computation. 


\section{Existence of a positive solution}

In this section we wish to deduce the existence of at least one positive solution to BVP (1.2). To accomplish this, we shall construct a subcone of $P$ as follows:

$$
Q=\left\{x \in P: x(t) \geq \frac{\Lambda}{H(1)} t(1-t)\|x\| \text { for } t \in[0,1]\right\} .
$$

For any $r>0$, let $Q_{r}=\{x \in Q:\|x\|<r\}, \partial Q_{r}=\{x \in Q:\|x\|=r\}, \bar{Q}_{r}=\{x \in Q:\|x\| \leq r\}$.

Lemma 4.1 ([33]) Let $Q$ be a cone in Banach space E. Suppose that $T: \bar{Q}_{r} \rightarrow Q$ is a completely continuous operator.

(i) If there exists $y_{0} \in Q \backslash\{\theta\}$ such that $y-T y \neq \mu y_{0}$ for any $y \in \partial Q_{r}$ and $\mu \geq 0$, then $i\left(T, Q_{r}, Q\right)=0$.

(ii) If $T y \neq \mu y$ for any $y \in \partial Q_{r}$ and $\mu \geq 1$, then $i\left(T, Q_{r}, Q\right)=1$.

Lemma 4.2 ([33]) Let $\Omega_{1}$ and $\Omega_{2}$ be two bounded open sets in Banach space E such that $\theta \in \Omega_{1}$ and $\bar{\Omega}_{1} \subset \Omega_{2}$. Let operator $T:\left(\bar{\Omega}_{2} \backslash \Omega_{1}\right) \cap Q \rightarrow Q$ is a completely continuous. Suppose that one of the two conditions

(i) if $T y \ngtr y, \forall y \in \partial Q \cap \Omega_{1} ; T y \not \leq y, \forall y \in \partial Q \cap \Omega_{2}$ and

(ii) if $T y \not \leq y, \forall y \in \partial Q \cap \Omega_{1} ; T y \ngtr y, \forall y \in \partial Q \cap \Omega_{2}$ is satisfied.

Then $T$ has at least one fixed point in $\left(\Omega_{2} \backslash \bar{\Omega}_{1}\right) \cap Q$.

Lemma 4.3 Assume that $\left(\mathrm{H}_{0}\right),\left(\mathrm{H}_{1}\right)$ and $\left(\mathrm{H}_{2}\right)$ hold, then $T: Q \rightarrow Q$ is completely continuous.

Proof For any $x \in Q, t \in[0,1]$, by Lemma 2.5 , we have

$$
\begin{aligned}
(T x)(t) & =\int_{0}^{1} K(t, s) a(s) f\left(s, s^{\alpha-2} x(s)\right) d s \\
& \leq H(1) \int_{0}^{1} s(1-s)^{\alpha-1} a(s) f\left(s, s^{\alpha-2} x(s)\right) d s .
\end{aligned}
$$

That is,

$$
\|T x\| \leq H(1) \int_{0}^{1} s(1-s)^{\alpha-1} a(s) f\left(s, s^{\alpha-2} x(s)\right) d s .
$$

On the other hand, by Lemma 2.5 , we also have

$$
\begin{aligned}
(T x)(t) & =\int_{0}^{1} K(t, s) a(s) f\left(s, s^{\alpha-2} x(s)\right) d s \\
& \geq \Lambda t(1-t) \int_{0}^{1} s(1-s)^{\alpha-1} a(s) f\left(s, s^{\alpha-2} x(s)\right) d s \\
& \geq \frac{\Lambda}{H(1)} t(1-t)\|T x\| .
\end{aligned}
$$

Therefore, $T(Q) \subset Q$. And, by a standard argument, we know that $T: Q \rightarrow Q$ is completely continuous. The proof is completed. 
Theorem 4.4 Suppose that the conditions $\left(\mathrm{H}_{0}\right),\left(\mathrm{H}_{1}\right)$ and $\left(\mathrm{H}_{2}\right)$ are satisfied, and

$$
\begin{aligned}
& \liminf _{y \rightarrow 0^{+}} \frac{f(t, y)}{y}>\lambda_{1}, \quad \text { uniformly on } t \in[0,1], \\
& \limsup _{y \rightarrow+\infty} \frac{f(t, y)}{y}<\lambda_{1}, \quad \text { uniformly on } t \in[0,1],
\end{aligned}
$$

where $\lambda_{1}$ is the first eigenvalue of $L$ defined by (2.3). Then BVP (1.2) has at least one positive solution.

Proof It follows from (4.1) that there exist $r>0$ and $\varepsilon_{1}>0$, such that

$$
f(t, y) \geq\left(\lambda_{1}+\varepsilon_{1}\right) y, \quad 0 \leq y \leq r, t \in[0,1] .
$$

Let $\varphi^{*}$ be the positive eigenfunction of $L$ corresponding to $\lambda_{1}$, thus $\varphi^{*}=\lambda_{1} L \varphi^{*}$. For any $x \in \partial Q_{r}$, by virtue of (2.2) and (4.3), we have

$$
(T x)(t) \geq\left(\lambda_{1}+\varepsilon_{1}\right) \int_{0}^{1} K(t, s) a(s) s^{\alpha-2} x(s) d s=\left(\lambda_{1}+\varepsilon_{1}\right)(L x)(t), \quad t \in[0,1] .
$$

We may suppose that $T$ has no fixed point on $\partial Q_{r}$ (otherwise, the proof is finished). Now we show that

$$
x-T x \neq \mu \varphi^{*}, \quad x \in \partial Q_{r}, \mu \geq 0 .
$$

If otherwise, there exist $x_{0} \in \partial Q_{r}$ and $\mu_{0} \geq 0$ such that $x_{0}-T x_{0}=\mu_{0} \varphi^{*}$. Obviously, $\mu_{0}>0$ and $x_{0}=T x_{0}+\mu_{0} \varphi^{*} \geq \mu_{0} \varphi^{*}$. Let $\bar{\mu}=\sup \left\{\mu \mid x_{0} \geq \mu \varphi^{*}\right\}$, then $\bar{\mu} \geq \mu_{0}>0$ and $x_{0} \geq \bar{\mu} \varphi^{*}$. Since $L(Q) \subset Q$, we have $\left(\lambda_{1}+\varepsilon_{1}\right) L x_{0} \geq \lambda_{1} L x_{0} \geq \lambda_{1} \bar{\mu} L \varphi^{*}=\bar{\mu} \varphi^{*}$. Therefore, using (4.4), we have

$$
x_{0}=T x_{0}+\mu_{0} \varphi^{*} \geq \lambda_{1} L x_{0}+\mu_{0} \varphi^{*} \geq \bar{\mu} \varphi^{*}+\mu_{0} \varphi^{*}=\left(\bar{\mu}+\mu_{0}\right) \varphi^{*},
$$

which contradicts the definition of $\bar{\mu}$. Hence (4.5) is true and it follows from Lemma 4.1 that

$$
i\left(T, Q_{r}, Q\right)=0 .
$$

Considering (4.2), there exist $R_{0}>r$ and $\varepsilon_{2}\left(0<\varepsilon_{2}<\lambda_{1}\right)$ such that

$$
f(t, y) \leq\left(\lambda_{1}-\varepsilon_{2}\right) y, \quad \forall y \geq R_{0}, t \in[0,1] .
$$

By the hypothesis $\left(\mathrm{H}_{1}\right)$, we have

$$
M_{0}=\max \left\{f(t, y) \mid t \in[0,1], 0 \leq y \leq R_{0}\right\}<+\infty .
$$

Hence

$$
f(t, y) \leq\left(\lambda_{1}-\varepsilon_{2}\right) y+M_{0}, \quad \text { for any } y \geq 0, t \in[0,1] .
$$


Now we set

$$
B=\{x \in Q, x=\mu T x, 0 \leq \mu \leq 1\}
$$

In the following, we shall show that $B$ is bounded. In fact, for any $x \in B, \mu \in[0,1]$, by (4.8), we have

$$
\begin{aligned}
x(t) & =\mu(T x)(t) \leq \int_{0}^{1} K(t, s) a(s) f\left(s, s^{\alpha-2} x(s)\right) d s \\
& \leq \int_{0}^{1} K(t, s) a(s)\left[\left(\lambda_{1}-\varepsilon_{2}\right) s^{\alpha-2} x(s)+M_{0}\right] d s \\
& =\left(\lambda_{1}-\varepsilon_{2}\right)(L x)(t)+M,
\end{aligned}
$$

where $M=M_{0} \sup _{t \in[0,1]} \int_{0}^{1} K(t, s) a(s) d s$. Since $r\left(\left(\lambda_{1}-\varepsilon_{2}\right) L\right)<1$, so $I-\left(\lambda_{1}-\varepsilon_{2}\right) L$ is reversible and

$$
\left(I-\left(\lambda_{1}-\varepsilon_{2}\right) L\right)^{-1}=I+\left(\lambda_{1}-\varepsilon_{2}\right) L+\left(\left(\lambda_{1}-\varepsilon_{2}\right) L\right)^{2}+\cdots+\left(\left(\lambda_{1}-\varepsilon_{2}\right) L\right)^{n}+\cdots
$$

From $L: P \rightarrow P$ we have $\left(I-\left(\lambda_{1}-\varepsilon_{2}\right) L\right)^{-1}(P) \subset P$. This together with (4.9) implies that

$$
x(t) \leq\left(I-\left(\lambda_{1}-\varepsilon_{2}\right) L\right)^{-1} M, \quad t \in[0,1]
$$

which shows that $B$ is bounded.

Take $R>\max \left\{R_{0}, \sup B\right\}$, we can easily obtain

$$
x \neq \mu T x, \quad x \in \partial Q_{R}, \mu \in[0,1] .
$$

This together with Lemma 4.1 yields

$$
i\left(T, Q_{R}, Q\right)=1 .
$$

It follows from (4.6) and (4.10) that

$$
i\left(T, Q_{R} \backslash \bar{Q}_{r}, Q\right)=i\left(T, Q_{R}, Q\right)-i\left(T, Q_{r}, Q\right)=1
$$

Then $T$ has at least one fixed point on $Q_{R} \backslash \bar{Q}_{r}$, which implies that BVP (1.2) has at least one positive solution. The proof is completed.

Now we consider another case of the problem (1.2). For this purpose, we define a linear operator $L_{\tau}$ for any sufficiently small $0<\tau<\frac{1}{2}$ as follows:

$$
\left(L_{\tau} x\right)(t)=\int_{\tau}^{1-\tau} K(t, s) a(s) s^{\alpha-2} x(s) d s, \quad t \in[0,1] .
$$

Obviously, from Lemma 2.7 we know that $L_{\tau}: E \rightarrow E$ is a $u_{0}$-bounded and positive operator with $r\left(L_{\tau}\right)>0$. Moreover $L_{\tau}$ has a positive eigenfunction $x_{\tau}$ corresponding to its first eigenvalue $\lambda_{\tau}=\left(r\left(L_{\tau}\right)\right)^{-1}$. 
Lemma 4.5 Suppose that $\left(\mathrm{H}_{0}\right)$ and $\left(\mathrm{H}_{1}\right)$ hold, there exists an eigenvalue $\tilde{\lambda}_{1}$ of $L$ such that

$$
\lim _{\tau \rightarrow 0^{+}} \lambda_{\tau}=\tilde{\lambda}_{1}
$$

Proof Take $\tau_{n} \in\left(0, \frac{1}{2}\right)(n=1,2, \ldots)$ such that $\tau_{1} \geq \tau_{2} \geq \cdots \geq \tau_{n} \geq \cdots$ and $\tau_{n} \rightarrow 0(n \rightarrow \infty)$. So for any $m>n$ and $x \geq \theta$, we have

$$
\left(L_{\tau_{n}} x\right)(t) \leq\left(L_{\tau_{m}} x\right)(t) \leq(L x)(t), \quad t \in[0,1]
$$

and

$$
\left(L_{\tau_{n}}^{k} x\right)(t) \leq\left(L_{\tau_{m}}^{k} x\right)(t) \leq\left(L^{k} x\right)(t), \quad t \in[0,1], k=2,3, \ldots
$$

where $L_{\tau_{n}}^{k}=L\left(L_{\tau_{n}}^{k-1}\right), k=2,3, \ldots$. Consequently, $\left\|L_{\tau_{n}}^{k}\right\| \leq\left\|L_{\tau_{m}}^{k}\right\| \leq\left\|L^{k}\right\|, k=1,2, \ldots$. From Gelfand's formula, we get $\lambda_{1} \leq \lambda_{\tau_{m}} \leq \lambda_{\tau_{n}}$, where $\lambda_{1}$ is the first eigenvalue of $L$. Since $\left\{\lambda_{\tau_{n}}\right\}$ is monotonous with lower boundedness $\lambda_{1}$, let

$$
\lim _{n \rightarrow \infty} \lambda_{\tau_{n}}=\tilde{\lambda}_{1}
$$

Now we shall show that $\tilde{\lambda}_{1}$ is an eigenvalue of $L$. Suppose $x_{\tau_{n}}$ is a positive eigenfunction of $L_{\tau_{n}}$ corresponding to $\lambda_{\tau_{n}}$ with $\left\|x_{\tau_{n}}\right\|=1, n=1,2, \ldots$, i.e.,

$$
x_{\tau_{n}}(t)=\lambda_{\tau_{n}} \int_{\tau_{n}}^{1-\tau_{n}} K(t, s) a(s) s^{\alpha-2} x_{\tau_{n}}(s) d s=\lambda_{\tau_{n}} L_{\tau_{n}} x_{\tau_{n}}(t), \quad t \in[0,1]
$$

It follows from the uniform continuity of the function $K(t, s)$ that $\left\{x_{\tau_{n}}\right\}$ is equicontinuous and uniformly bounded. By the Arzela-Ascoli theorem, $\left\{x_{\tau_{n}}\right\}$ has a subsequence which converges to some $\widetilde{x}_{0}$ as $n \rightarrow \infty$. Without loss of generality, we may assume $x_{\tau_{n}} \rightarrow \widetilde{x}_{0}$ as well. Obviously, $\widetilde{x}_{0} \geq \theta$ and $\left\|\widetilde{x}_{0}\right\|=1$.

By (4.11), we have

$$
\tilde{x}_{0}(t)=\widetilde{\lambda}_{1} \int_{0}^{1} K(t, s) a(s) s^{\alpha-2} \widetilde{x}_{0}(s) d s, \quad t \in[0,1]
$$

that is, $\widetilde{x}_{0}=\tilde{\lambda}_{1} L \widetilde{x}_{0}$ and $\tilde{\lambda}_{1}$ is the eigenvalue of the operator $L$. The proof is completed.

Theorem 4.6 Suppose that the conditions $\left(\mathrm{H}_{0}\right),\left(\mathrm{H}_{1}\right)$ and $\left(\mathrm{H}_{2}\right)$ are satisfied, and

$$
\begin{aligned}
& \limsup _{y \rightarrow 0^{+}} \frac{f(t, y)}{y}<\lambda_{1}, \quad \text { uniformly on } t \in[0,1], \\
& \liminf _{y \rightarrow+\infty} \frac{f(t, y)}{y}>\tilde{\lambda}_{1}, \quad \text { uniformly on } t \in[0,1]
\end{aligned}
$$

where $\lambda_{1}, \tilde{\lambda}_{1}$ are the eigenvalues of $L$ and $\lambda_{1}$ is the first eigenvalue of $L$. Then $B V P(1.2)$ has at least one positive solution. 
Proof It follows from (4.12) that there exist $\bar{r}>0$ and $\varepsilon_{3}\left(0<\varepsilon_{3}<\lambda_{1}\right)$ such that

$$
f(t, y) \leq\left(\lambda_{1}-\varepsilon_{3}\right) y, \quad 0 \leq y \leq \bar{r}, t \in[0,1] .
$$

Now we show that

$$
T x \not x, \quad x \in \partial Q_{\bar{r}} .
$$

Otherwise, there exists $v_{0} \in \partial Q_{\bar{r}}$ such that $v_{0} \leq T v_{0}$. Then we have

$$
0 \leq v_{0}(t) \leq\left(T v_{0}\right)(t), \quad \forall t \in[0,1]
$$

By (4.16) and (4.14), we have

$$
\begin{aligned}
v_{0}(t) & \leq \int_{0}^{1} K(t, s) a(s) f\left(s, s^{\alpha-2} v_{0}(s)\right) d s \\
& \leq\left(\lambda_{1}-\varepsilon_{3}\right) \int_{0}^{1} K(t, s) a(s) s^{\alpha-2} v_{0}(s) d s=\left(\lambda_{1}-\varepsilon_{3}\right)\left(L v_{0}\right)(t),
\end{aligned}
$$

i.e.

$$
\nu_{0} \leq\left(\lambda_{1}-\varepsilon_{3}\right)\left(L v_{0}\right)
$$

Acting $L$ on (4.17) $n-1$ times, we obtain

$$
v_{0}(t) \leq\left(\lambda_{1}-\varepsilon_{3}\right)^{n}\left(L^{n} v_{0}\right)(t)
$$

So we have

$$
\left\|L^{n}\right\| \geq \frac{\left\|L^{n} v_{0}\right\|}{\left\|v_{0}\right\|} \geq \frac{1}{\left(\lambda_{1}-\varepsilon_{3}\right)^{n}}, \quad n=1,2, \ldots
$$

By Gelfand's formula, we know

$$
r(L)=\lim _{n \rightarrow \infty}\left\|L^{n}\right\|^{\frac{1}{n}} \geq \lim _{n \rightarrow \infty}\left(\frac{1}{\left(\lambda_{1}-\varepsilon\right)^{n}}\right)^{\frac{1}{n}}>\frac{1}{\lambda_{1}-\varepsilon_{3}}>\frac{1}{\lambda_{1}},
$$

which is a contradiction with $r(L)=\lambda_{1}^{-1}$. So (4.15) holds.

It follows from (4.13) that there exist $\varepsilon_{4}\left(0<\varepsilon_{4}<\tilde{\lambda}_{1}\right)$ and $R_{0}>1$ such that

$$
f(t, y) \geq\left(\tilde{\lambda}_{1}+\varepsilon_{4}\right) y, \quad y \geq R_{0}, t \in[0,1] .
$$

From the proof of Lemma 4.5, there exists $\tau \in\left(0, \frac{1}{2}\right)$ such that $\tilde{\lambda}_{1} \leq \lambda_{\tau}=\left(r\left(L_{\tau}\right)\right)^{-1}<\tilde{\lambda}_{1}+$ $\varepsilon_{4}$. Take $\bar{R}=\max \left\{R_{0}, \frac{R_{0} H(1)}{\Lambda \tau^{2}}\right\}$. We shall check that

$$
T x \not x, \quad x \in \partial Q_{\bar{R}} .
$$


If it is not true, there exists $\phi \in \partial Q_{\bar{R}}$ such that $T \phi \leq \phi$. Then it follows from the construction of $Q$ that

$$
\phi(t) \geq \frac{\Lambda t(1-t)}{H(1)}\|\phi\| \geq \frac{\Lambda \tau(1-\tau)}{H(1)} \bar{R} \geq \frac{\Lambda \tau^{2}}{H(1)} \bar{R} \geq R_{0}, \quad t \in[\tau, 1-\tau] .
$$

Thus, by (4.18) we have

$$
\begin{aligned}
\phi(t) & \geq(T \phi)(t)=\int_{0}^{1} K(t, s) a(s) f\left(s, s^{\alpha-2} \phi(s)\right) d s \\
& \geq \int_{\tau}^{1-\tau} K(t, s) a(s) f\left(s, s^{\alpha-2} \phi(s)\right) d s \\
& \geq\left(\tilde{\lambda}_{1}+\varepsilon_{4}\right) \int_{\tau}^{1-\tau} K(t, s) a(s) s^{\alpha-2} \phi(s) d s \\
& =\left(\tilde{\lambda}_{1}+\varepsilon_{4}\right)\left(L_{\tau} \phi\right)(t) .
\end{aligned}
$$

Notice that $\phi \geq \theta$, by Lemma 2.10, there exists $\mu \geq 0$ such that $\phi=\mu x_{\tau}, x_{\tau}$ is the positive eigenfunction corresponding to the first eigenvalue $\lambda_{\tau}$ of the operator $L_{\tau}$. If $\mu=0$, then $\phi(t) \equiv 0, t \in[0,1]$. If $\mu>0$, by (4.20), we know that $\mu x_{\tau}(t) \geq\left(\widetilde{\lambda}_{1}+\varepsilon_{4}\right)\left(L_{\tau} \phi\right)(t)=\mu\left(\tilde{\lambda}_{1}+\right.$ $\left.\varepsilon_{4}\right)\left(L_{\tau} x_{\tau}\right)(t)$, i.e. $x_{\tau} \geq\left(\tilde{\lambda}_{1}+\varepsilon_{4}\right) L_{\tau} x_{\tau}$. Considering $\lambda_{\tau}<\tilde{\lambda}_{1}+\varepsilon_{4}$, we have $x_{\tau}=\lambda_{\tau} L_{\tau} x_{\tau}<\left(\tilde{\lambda}_{1}+\right.$ $\left.\varepsilon_{4}\right) L_{\tau} x_{\tau}$, a contradiction. Thus, (4.19) is true. By (4.15), (4.19) and Lemma 4.2, we know that $T$ has a positive fixed point in $Q_{\bar{R}} \backslash \bar{Q}_{\bar{r}}$, which implies that BVP (1.2) has at least one positive solution. The proof is completed.

Example 4.7 Consider the following problem:

$$
\left\{\begin{array}{l}
-D_{0+}^{\frac{3}{2}} x(t)+\frac{1}{5} x(t)=a(t) f(t, x(t)), \quad 0<t<1, \\
x(0)=0, \quad x(1)=0 .
\end{array}\right.
$$

Here

$$
a(t)=\frac{1}{\sqrt[4]{t} \sqrt[8]{(1-t)^{3}}}, \quad t \in(0,1), \quad f(t, y)=\sqrt{y}+\ln (1+y), \quad(t, y) \in[0,1] \times[0, \infty) .
$$

Obviously, $f:[0,1] \times[0, \infty) \rightarrow[0, \infty)$ is continuous, $a:(0,1) \rightarrow[0, \infty)$ is continuous and $a(t)$ is singular at $t=0$ and $t=1$. Moreover,

$$
\begin{aligned}
0 & <\int_{0}^{1} s^{\alpha-1}(1-s)^{\alpha-1} a(s) d s=\int_{0}^{1} s^{\frac{3}{2}-1}(1-s)^{\frac{3}{2}-1} \frac{1}{\sqrt[4]{s} \sqrt[8]{(1-s)^{3}}} d s \\
& =\int_{0}^{1} s^{\frac{5}{4}-1}(1-s)^{\frac{9}{8}-1} d s=B\left(\frac{5}{4}, \frac{9}{8}\right)<+\infty, \\
0 & <\int_{0}^{1} s^{\alpha-2}(1-s)^{\alpha-2} a(s) d s=\int_{0}^{1} s^{\frac{3}{2}-2}(1-s)^{\frac{3}{2}-2} \frac{1}{\sqrt[4]{s} \sqrt[8]{(1-s)^{3}}} d s \\
& =\int_{0}^{1} s^{\frac{1}{4}-1}(1-s)^{\frac{1}{8}-1} d s=B\left(\frac{1}{4}, \frac{1}{8}\right)<+\infty,
\end{aligned}
$$

where $B(\cdot, \cdot)$ denotes a beta function. 
Noticing that $\Gamma(\cdot)$ is strictly increasing on $[2, \infty)$, so for any $y \in[0, \infty)$ we have

$$
\begin{aligned}
h(y) & =\frac{\alpha-2}{\Gamma(\alpha-1)}+\sum_{k=1}^{+\infty} \frac{y^{k}}{\Gamma((k+1) \alpha-2)}=-\frac{1}{2 \sqrt{\pi}}+\sum_{k=1}^{+\infty} \frac{y^{k}}{\Gamma\left(\frac{3}{2} k-\frac{1}{2}\right)} \\
& =-\frac{1}{2 \sqrt{\pi}}+y+\sum_{k=2}^{+\infty} \frac{y^{k}}{\Gamma\left(\frac{3}{2} k-\frac{1}{2}\right)} \leq-\frac{1}{2 \sqrt{\pi}}+y+\sum_{k=2}^{+\infty} \frac{y^{k}}{\Gamma(k)} \\
& =-\frac{1}{2 \sqrt{\pi}}+y\left(1+\sum_{k=2}^{+\infty} \frac{y^{k}}{\Gamma(k)}\right)=-\frac{1}{2 \sqrt{\pi}}+y e^{y} .
\end{aligned}
$$

By direct calculation, we have

$$
\frac{1}{2 \sqrt{\pi}} \approx 0.282095, \quad \frac{1}{5} e^{\frac{1}{5}} \approx 0.244281
$$

(4.22) implies that $h\left(\frac{1}{5}\right)<0$, combining with the monotonicity of $h(y)$, we can obtain $\frac{1}{5}<\tilde{b}$ $(\tilde{b}$ is unique zero point of function $h$, i.e., $h(\tilde{b})=0$ ).

On the other hand, it is obvious that

$$
\begin{aligned}
& \liminf _{y \rightarrow 0^{+}} \frac{f(t, y)}{y}=\liminf _{y \rightarrow 0^{+}} \frac{\sqrt{y}+\ln (1+y)}{y}=+\infty, \\
& \limsup _{y \rightarrow+\infty} \frac{f(t, y)}{y}=\limsup _{y \rightarrow+\infty} \frac{\sqrt{y}+\ln (1+y)}{y}=0,
\end{aligned}
$$

uniformly on $t \in[0,1]$, which implies that

$$
\limsup _{y \rightarrow+\infty} \frac{f(t, y)}{y}<\lambda_{1}<\liminf _{y \rightarrow 0^{+}} \frac{f(t, y)}{y} .
$$

Therefore, all conditions of Theorem 4.4 are satisfied. Thus Theorem 4.4 ensures that BVP (4.21) has at least one positive solution.

Remark 4.8 In BVP (4.21), note that $a(t)$ is singular at $t=0$ and $t=1$, that is to say, the nonlinearity has singularity at $t=0$ and $t=1$. This is in essence different from a Dirichlettype BVP $[4,10,13]$.

\section{Acknowledgements}

The authors would like to thank the referee for his/her very important comments, which improved the results and the quality of the paper. This work was supported financially by Project funded by China Postdoctoral Science Foundation (2017M612231), Doctoral Scientific Research Foundation of Qufu Normal University and Youth Foundation of Qufu Normal University (BSQD20130140, XKJ201303).

\section{Competing interests}

The authors declare that they have no competing interests.

\section{Authors' contributions}

All authors contributed equally to the writing of this paper. All authors read and approved the final manuscript.

\section{Publisher's Note}

Springer Nature remains neutral with regard to jurisdictional claims in published maps and institutional affiliations. 


\section{References}

1. Agarwal, R.P., O'Regan, D., Staněk, S.: Positive solutions for Dirichlet problems of singular nonlinear fractional differential equations. J. Math. Anal. Appl. 371, 57-68 (2010)

2. Ahmad, B.: Sharp estimates for the unique solution of two-point fractional-order boundary value problems. Appl. Math. Lett. 65, 77-82 (2017)

3. Bai, Z.: Solvability for a class of fractional $m$-point boundary value problem at resonance. Comput. Math. Appl. 62 1292-1302 (2011)

4. Bai, Z., Lü, H.: Positive solutions for boundary value problem of nonlinear fractional differential equation. J. Math. Anal. Appl. 311, 495-505 (2005)

5. Cabada, A., Kisela, T.: Existence of positive periodic solutions of some nonlinear fractional differential equations. Commun. Nonlinear Sci. Numer. Simul. 50, 51-67 (2017)

6. Caballero, J., Harjani, J., Sadarangani, K.: Positive solutions for a class of singular fractional boundary value problems. Comput. Math. Appl. 62, 1325-1332 (2011)

7. Cui, Y.: Uniqueness of solution for boundary value problems for fractional differential equations. Appl. Math. Lett. 51, 48-54 (2016)

8. Goodrich, C.: Existence and uniqueness of solutions to a fractional difference equation with nonlocal conditions. Comput. Math. Appl. 61, 191-202 (2011)

9. Guo, L., Liu, C.: Existence of positive solutions for singular higher-order fractional differential equation via spectral analysis. J. Appl. Math. Comput. 54, 357-379 (2017)

10. Han, X., Gao, H.: Existence of positive solutions for eigenvalue problem of nonlinear fractional differential equations Adv. Differ. Equ. 2012, 66 (2012)

11. Hao, X., Liu, L., Wu, Y.: Positive solutions for nonlinear fractional semipositone differential equation with nonlocal boundary conditions. J. Nonlinear Sci. Appl. 9, 3992-4002 (2016)

12. Jebari, R.: Solvability and positive solutions of a system of higher order fractional boundary value problem with integral conditions. Fract. Differ. Calc. 6, 179-199 (2016)

13. Jiang, D., Yuan, C.: The positive properties of Green's function for Dirichlet-type boundary value problems of nonlinear fractional differential equations and its application. Nonlinear Anal. 72, 710-719 (2010)

14. Jiang, J., Liu, L.: Existence of solutions for a sequential fractional differential system with coupled boundary conditions. Bound. Value Probl. 2016, 159 (2016)

15. Jiang, J., Liu, L., Wu, Y.: Positive solutions to singular fractional differential system with coupled boundary conditions. Commun. Nonlinear Sci. Numer. Simul. 18, 3061-3074 (2013)

16. Jiang, J., Liu, L., Wu, Y.: Multiple positive solutions of singular fractional differential system involving Stieltjes integral conditions. Electron. J. Qual. Theory Differ. Equ. 2012, 43 (2012)

17. Jiang, J., Liu, L., Wu, Y.: Positive solutions for nonlinear fractional differential equations with boundary conditions involving Riemann-Stieltjes integrals. Abstr. Appl. Anal. 2012, Article ID 708192 (2012)

18. Jiang, W.: Solvability of fractional differential equations with $p$-Laplacian at resonance. Appl. Math. Comput. 260 48-56 (2015)

19. Kilbas, A.A., Srivastava, H.M., Trujillo, J.J.: Theory and Applications of Fractional Differential Equations. North-Holland Mathematics Studies. Elsevier, Amsterdam (2006)

20. Liu, L., Li, H., Liu, C., Wu, Y.: Existence and uniqueness of positive solutions for singular fractional differential systems with coupled integral boundary conditions. J. Nonlinear Sci. Appl. 10, 243-262 (2017)

21. Podlubny, I.: Fractional Differential Equations. An Introduction to Fractional Derivatives, Fractional Differential Equations, to Methods of Their Solution and Some of Their Applications. Mathematics in Science and Engineering. Academic Press, San Diego (1999)

22. Reutskiy, S.: A new numerical method for solving high-order fractional eigenvalue problems. J. Comput. Appl. Math. 317, 603-623 (2017)

23. Wang, Y., Jiang, J.: Existence and nonexistence of positive solutions for the fractional coupled system involving generalized p-Laplacian. Adv. Differ. Equ. 2017, 337 (2017)

24. Wang, Y., Liu, L., Wu, Y.: Positive solutions for singular semipositone boundary value problems on infinite intervals. Appl. Math. Comput. 227, 256-273 (2014)

25. Wang, Y., Liu, L.: Positive solutions for a class of fractional 3-point boundary value problems at resonance. Adv. Differ Equ. 2017, 7 (2017)

26. Wang, Y., Liu, L.: Positive properties of the Green function for two-term fractional differential equations and its application. J. Nonlinear Sci. Appl. 10, 2094-2102 (2017)

27. Xu, X., Jiang, D., Yuan, C.: Multiple positive solutions to singular positone and semipositone Dirichlet-type boundary value problems of nonlinear fractional differential equations. Nonlinear Anal. 74, 5685-5696 (2011)

28. Zhang, X., Liu, L., Wu, Y.: Multiple positive solutions of a singular fractional differential equation with negatively perturbed term. Math. Comput. Model. 55, 1263-1274 (2012)

29. Zhang, X., Liu, L., Wu, Y., Wiwatanapataphee, B.: The spectral analysis for a singular fractional differential equation with a signed measure. Appl. Math. Comput. 257, 252-263 (2015)

30. Zou, Y., He, G.: On the uniqueness of solutions for a class of fractional differential equations. Appl. Math. Lett. 74 68-73 (2017)

31. Krasnosel'skii, M.A.: Positive Solutions of Operator Equations. Noordhoff, Groningen (1964)

32. Deimling, K.: Nonlinear Functional Analysis. Springer, New York (1985)

33. Guo, D., Lakshmikantham, V.: Nonlinear Problems in Abstract Cone. Academic Press, New York (1988) 